Théologiques

Théologiques

\title{
Territoire et sacré chez les Innus
}

\section{Anne Doran}

Volume 16, numéro 1, 2008

Le territoire et le sacré

URI : https://id.erudit.org/iderudit/019187ar

DOI : https://doi.org/10.7202/019187ar

Aller au sommaire du numéro

\section{Éditeur(s)}

Faculté de théologie et de sciences des religions, Université de Montréal

\section{ISSN}

1188-7109 (imprimé)

1492-1413 (numérique)

Découvrir la revue

\section{Citer cet article}

Doran, A. (2008). Territoire et sacré chez les Innus. Théologiques, 16(1), 117-142. https://doi.org/10.7202/019187ar

\section{Résumé de l'article}

À partir d'une comparaison entre l'attitude de l'Occidental face au monde qu'il considère avant tout comme un objet d'exploitation - et celle de l'Innu qui le perçoit comme un tout qui le porte et à l'intérieur duquel il doit se situer en relation d'alliance avec les autres éléments qui le constituent, cet article examine les fondements de la conception innue qui sont à l'origine de cette attitude : un monde dont chacun des éléments dévoile le sacré et à l'intérieur duquel tous les groupes d'êtres entretiennent des rapports de don. Il en conclut que pour les Innus, l’identité est liée au territoire. L'état québécois comprendra-t-il qu'une assise territoriale s'avère nécessaire à l'existence même de ce peuple et saurons-nous profiter de l'apport que sa conception pourrait apporter aux questions environnementales? 


\title{
Territoire et sacré chez les Innus
}

\author{
Anne DORAN* \\ Département des sciences humaines \\ Université du Québec à Chicoutimi
}

Les deux responsables innues du Musée de Mashteuiatsh me disaient lorsque j'y suis allée, il y a quelques années, que pour saisir la spiritualité innue il me faudrait suivre un groupe familial sur les territoires plutôt que de rester au village. Au village, je trouverais la religion; mais pour les questions de spiritualité, il me fallait accompagner des Innus sur les territoires — et cela sous-entend évidemment les territoires de chasse. Qu'est-ce que cette distinction entre village et territoires et entre religion et spiritualité - cette dernière comportant de toute évidence une connotation innue particulière - pouvait bien signifier? À quelle réalité culturelle ces femmes faisaient-elles allusion? La forêt et la chasse étaient prégnantes d'un sens qu'on ne retrouvait pas dans la vie sédentaire du village, celle-ci ressemblant fort à la vie des habitants blancs de la ville de Roberval, située juste à côté de la réserve. Qu'est-ce qui lie l'Innu à son territoire ancestral et qu'il ne retrouve pas ailleurs dans l'espace sédentarisé du village? Quel lien porteur de spiritualité l'unit à son territoire de telle sorte que c'est là, en le parcourant pour chasser, qu'il reprendra contact avec un sens du spirituel que la religion au village — religion à laquelle ses ancêtres se sont pourtant

\footnotetext{
Anne Doran est professeur associé au Département des Sciences humaines de l'Université du Québec à Chicoutimi. Elle travaille depuis plusieurs années sur les liens entre le christianisme reçu par les Innus et leur culture traditionnelle. Elle a publié en 2005 à L'Harmattan un livre - Spiritualité traditionnelle et christianisme chez les Montagnais - qui s'appuie sur une analyse des prières en langue montagnaise du Livre de prières et de chants que les différentes communautés de la Côte Nord du Saint-Laurent utilisent encore aujourd'hui. Ses recherches actuelles portent sur le don, élément fondamental de la compréhension traditionnelle du monde de ce peuple et qui permit à ce dernier de saisir le christianisme en le situant en continuité avec sa culture propre et en élaborant une interprétation originale.
} 
rapidement convertis - ne peut extérioriser de la même manière ? Car s'il ne s'agissait que d'une façon de discourir sur ce qui l'unit à ses territoires de chasse et qui est porteur de spiritualité, sans doute pourrait-il l'expliquer hors contexte. Et celui qui cherche à comprendre ce lien n'aurait pas besoin de prendre avec lui le chemin des bois. Mais comme il s'agit avant tout de ce qu'Ingold $(2000,168)$ nomme le dwelling, c'est-à-dire une attitude où la compréhension du vécu s'accomplit à l'intérieur même de l'action qui la suscite, le rôle du territoire dans l'identité et la spiritualité innues sont perceptibles dans la mise en œuvre des rapports mêmes qui définissent le lien de l'Innu à son territoire.

\section{Une identité fondée sur le lien à un territoire}

Pour l'Occidental, bien que quelques philosophes aient produit des réflexions qui tenaient la relation au monde comme essentielle à la compréhension de l'être humain — pensons ici à l'être-au-monde de Heiddegger (1966, 133) ou à la Phénoménologie de la perception de Merleau-Ponty (1945, 160-173) —, le monde se situe comme le vis-à-vis de l'humain et celui-ci le perçoit en s'y opposant. L'humain appréhende le monde comme un objet détaché de lui et jamais il ne lui viendrait à l'idée de l'envisager comme une réalité qui l'englobe et ainsi de se concevoir lui-même comme une de ses composantes. Il perçoit plutôt le monde comme ce sur quoi il a à agir pour y mettre en action les moyens techniques qu'il a développés et y poursuivre sa course infinie vers l'avancée du progrès. Pour la culture occidentale prise en bloc, le monde est essentiellement un objet à dominer ou dans le meilleur cas, à aménager. Une informatrice innue me faisait d'ailleurs remarquer que les Blancs éprouvaient le besoin d'agencer leur terrain conformément à leur goût — avec fleurs, arbres, voire plans d'eau ou rocailles - alors que pour les Innus, il était beaucoup plus normal de prendre la nature telle qu'elle se présentait et de la laisser être elle-même, en l'état. Le Blanc adapte le territoire à ses besoins et le change selon l'orientation de ses projets. Dans sa réflexion sur le désenchantement du monde, où il décrit le passage d'une conception où visible/invisible sont inclus dans les différents éléments du monde - comme c'est le cas dans la conception innue du monde - à celui d'une transcendance créatrice séparée de lui avec entre autres l'avènement du christianisme -, Gauchet décrit le changement qui en résulte comme « refus fondamental du donné immédiat qui [...] pousse à agir sur lui, qui [...] induit sa négation active - doublement, donc, sous forme d'une entreprise assimilatrice visant à le surmonter et à 
le réduire comme donné autre, et sous forme d'une entreprise transformatrice destinée à la produire comme autre qu'il n'est donné » $(1985,98)$. Quand on sait qu'une compagnie minière (Barrick Gold; projet Pascua Lama) envisage de déplacer, voire de supprimer un glacier pour exploiter l'or qui se trouve dessous au Chili, on ne trouve pas la description outrée...

L'Innu respecte l'ordre de la nature et cherche à s'y ajuster. Pour l'humain occidental, la nature a perdu sa capacité de révélation d'un autre ordre que celui du visible, d'une présence autre que celle de sa pure matérialité. Mais l'Innu ne peut se faire ni à cette banalisation de son environnement, ni à une exploitation qui n'ait pas sa contrepartie en termes de valorisation. Comment expliquer cette différence de perception?

Si l'analyse innue du réel, dans son lien actuel avec la culture occidentale, a pu changer sous certains aspects - elle a dû par exemple s'adapter à un vocabulaire juridique étranger à l'esprit de sa culture dans ses revendications territoriales (Lacasse) - , elle n'en demeure pas moins marquée par la perception traditionnelle du monde. Quels sont les traits qui caractérisent la culture innue actuelle dans son rapport avec le territoire? C'est au monde, à leur monde et à tout le lien que l'Innu entretient avec lui, à l'intérieur duquel sa vie trouve sens, là où s'enracine son identité et son sentiment d'appartenance à une communauté, que renvoie le lien au territoire. Qu'est-ce à dire?

Dans la conception innue du monde, l'humain se situe dans une appartenance à une réalité cosmique plus large que lui et à laquelle il cherche à s'intégrer. C'est dans un monde qui fait sens dans sa globalité que l'humain peut discerner qui il est et comment il doit agir. Il perçoit donc son existence comme liée à la nature qui l'environne. Il s'appréhende comme une réalité portée par le monde qui l'entoure tout autant qu'il envisage le monde comme une réalité qui appartient à son être propre en ce qu'elle est essentielle à sa vie, à son activité, au sens qu'il donne à son action. Dans un des documentaires d'Arthur Lamothe, des Innus nous disent pourquoi ils préfèrent vivre sous la tente plutôt que dans une maison et une des raisons qu'ils invoquent tient au fait que dans la maison, ils n'ont rien à faire (Lamothe, et al. 1978): la vie dans une maison est pour eux dépourvue de sens. De même, quand nous avons participé au Rassemblement des aînés auquel la communauté de Betsiamites convoquait les aînés de toutes les autres communautés innues de la Côte-Nord, pendant l'été 2000, un campement avait été organisé dans la forêt adjacente au village. En rencontrant les gens, nous avons été surprise de constater à quel point ils s'étaient installés 
confortablement en plein air, agissant avec la tente et ce qui en dépendait — abri et installations diverses - comme s'il s'agissait d'une véritable maison. La nature est leur demeure et c'est par plaisir qu'on voudra camper dans la forêt. Comme le souligne Mathieu Mestokosho, contrairement au Blanc que la moindre chose inquiète, l'Indien lui est à l'aise en forêt (Bouchard 2007, 100). De même, pour le pèlerinage et la célébration de la fête de sainte Anne aux Ilets-Jérémie, certaines familles iront s'installer sous la tente, sur les lieux mêmes, pour la durée de la neuvaine: le pèlerinage implique l'imprégnation du lien au territoire. Le rapport de l'Innu au monde n'en est pas un de domination - domination de l'humain sur la nature pour la plier à sa volonté et à son utilisation débridée - mais plutôt d'inclusion et de respect. C'est parce qu'il entretient une relation profonde avec la nature qui l'entoure, parce qu'il se trouve porté et soutenu par elle, qu'il s'y sent chez lui.

Pour les Innus, le groupe humain constitue un tout en lien avec les différentes entités qui l'environnent et l'espace qui le soutient. Le milieu de vie constitue une totalité dans laquelle l'humain s'insère en état de fraternité et d'égalité avec les autres éléments qui le composent; il est partie prenante de l'être même du groupe humain en ce qu'il participe activement à son mode d'exister, à ce qui le définit dans son être-au-monde (Heidegger 1966, 132). L'humain ne prend sa véritable dimension que dans sa relation avec d'autres êtres non-humains dans une nature qui le porte et lui permet de subsister. Il a conscience de ne pouvoir participer à la vie que dans sa relation à un tout qui le soutient dans l'être. Cet élément de référence à ce qui l'environne et forme son milieu de vie appartient à son être le plus personnel. C'est cela même qui le constitue. Il se perçoit comme humain à l'intérieur du tout où il a un statut spécifique. Le nom qu'il donne à son groupe est d'ailleurs significatif de cette façon de percevoir les choses: Innu, Innuat, l'humain, les êtres humains. Il peut se situer comme humain en référence à son appartenance à un ensemble d'êtres dont il se distingue, mais auquel il doit se référer pour pouvoir s'affirmer dans son être propre. Il n'y a de groupe humain que dans un rapport à tout ce qui existe et qui constitue le cosmos en tant que réalité englobant tous les êtres et les supportant tous. C'est l'ensemble qui fait sens et c'est en se situant dans l'ensemble que l'être humain définit son rôle et sait ce qu'il a à être: un élément du grand tout fraternel qu'il constitue avec la communauté des êtres. Il ne situe pas ce qui l'entoure comme une nature qui lui fait face et qu'il cherche à dominer ou à laquelle il s'oppose. Au contraire, il trouve sa place à l'intérieur d'une 
réalité qui lui fournit la clé de ce qu'il doit être en l'insérant dans un ensemble global: celui de la multiplicité des êtres qui appartiennent au cosmos.

Le territoire a une valeur telle parce qu'il fait vivre l'Innu. Mani Michel, une aînée algonquine de la bande du Grand lac Victoria - mais l'exemple vaut aussi pour les Innus - explique ainsi à son petit-fils : "Tous les anciens le disaient: "N'abandonnez jamais les lieux où l'on mange, les lieux où l'on vit” "(Leroux 1992, 34). Le lieu qui procure la vie à l'humain ne joue pas par rapport à lui un rôle aléatoire: nul autre ne lui est substituable parce que sa vie lui est liée. Le territoire où se déroulent les activités du groupe de chasse est perçu sous l'angle de l'abondance et de la générosité d'une nature qui donne à l'humain tout ce qu'il lui faut pour vivre: "Arrivés sur notre territoire, nous étions fiers d'avoir sous la main tout ce dont nous avions besoin pour vivre» (André 1984, 43). Ce sentiment de l'abondance des ressources qui sont disponibles et s'offrent généreusement à l'humain est à l'origine de la spontanéité du partage entre les membres de la communauté et même envers ceux de groupes voisins. La générosité de la nature sous-tend la solidarité avec tous ceux qui se présentent et auxquels on est à même de donner :

Quoique assez obscure pour les non-initiés, cette méthode (la poursuite du caribou en canot) était considérée comme le fer de lance de nos chasses, autrefois. Elle permettait d'amasser une grande quantité de viande fraîche. Ceci peut se comparer à un cultivateur blanc qui fait grande provision de ses produits au cours de l'été et en alimente le marché toute l'année. Il en était de même pour nous: on venait de partout s'approvisionner en viande, selon ses besoins [...] Certains venaient à la «Rivière Georges» avec toute leur famille. Quelle grande solidarité se manifestait alors! [...] il fallait non seulement tuer mais aussi préparer les prises afin que la viande ne s'avariât pas car elle était destinée à la communauté. Voici pourquoi ces chasseurs restaient en permanence à cet endroit, sans redescendre sur la côte. Cette entraide avait quelque chose d'extraordinaire et je pense, sans être le seul, que c'était là notre plus belle marque de fraternité! [...] Ces chasseurs, en agissant ainsi, avaient pour but d'empêcher la famine [...] Ils pourvoyaient à tous pour tout l'hiver! (André 1984, 117)

Le territoire se présente ainsi comme un bien commun, capable de faire vivre tous ceux qui s'en remettent à lui : "Autrefois, que nous soyons de "Sept-Îles" ou de "Goose Bay" nous chassions ensemble, parfois même arrivaient des trappeurs anglais [...] et nous n'avions jamais eu de dispute à propos du territoire au Labrador: tout le monde comprenait que chacun y gagnait sa vie» (André 1984, 117). 
Mais l'humain qui comprend ainsi son être propre dans le rapport au cosmos qui l'entoure - et qui se présente pour lui sous la forme du territoire - nourrit avec le monde un rapport de responsabilité ${ }^{1}$. Ce monde qui lui fournit tous les éléments dont il a besoin pour vivre, il doit en retour le respecter et le soigner.

Le respecter d'abord. L'admirer: "nous étions heureux dans notre milieu, dans l'immensité de nos terres, de la beauté des montagnes, des rivières et des lacs» (André 1984, 43). En respecter l'ordonnance naturelle: "Ils ont inondé nos habitats et noyé plusieurs beaux endroits. Ils enlevèrent la vie aux arbres en commençant à les couper dès qu'ils furent débarqués. Aujourd'hui, j'en éprouve de la pitié. Là-bas, en arrière, tout est bouleversé et le paysage n'a plus la même apparence " (Leroux 1992, 35). Éprouver de la gratitude face à cette abondance qui est mise à la disposition de l'humain, se sentir riche de la multiplicité de ce qui est offert:

Un vieil homme dit à son petit-fils: «[...] c'est à toi que je laisserai tout mon avoir». L'enfant regarde son grand-père, étonné. Il ne possède rien [...], penset-il. "Qu'est-ce que tu vas me laisser, grand-père ? " Et le vieux lui répond: «L'ensemble de mon territoire avec tout ce qui s'y trouve. Toutes les sortes d'animaux, toutes les sortes de poissons, les arbres, les rivières, voilà ce que je te laisse en héritage. De génération en génération, c'est de cela dont tu dépendras pour vivre. » (Lacasse 2004, 83 ; Kapesh 1979, 23).

L'Innu doit aussi soigner la terre qui lui donne vie: «Ils prenaient soin de la terre alors; ils veillaient sur elle.» Et constat du résultat de l'actuelle dépossession du territoire: "Aujourd'hui, les territoires sont à l'abandon. » Ou encore: «La terre est laissée sans soins» (Leroux 1992, 35). Les exemples de ce soin apporté à la préservation du territoire et de ses ressources seraient nombreux. Ainsi, Mathieu André écrit (1984, 44) : "Jadis, nous veillions à préserver nos territoires du feu, nous ne tuions pas sans raison les animaux, nous surveillions la gestation et la croissance normale du gibier, nous attendions pour chasser qu'il ait atteint un certain degré de développement». Mathieu André parle de ceux qui habitent en permanence les territoires environnant la Rivière Georges où se pratiquait la chasse aux caribous en canot dans les termes mêmes qui définissent ceux qui, dans notre culture, contrôlent la prise du gibier:

1. Le territoire peut nous sembler immense de l'extérieur quand nous pensons, par exemple, au périple annuel d'un Mathieu Mestokosho (Bouchard 1977) qui partait de Mingan pour se rendre à North-West River et revenir. Il est pourtant connu dans ses moindres détails, ainsi que l'ensemble des ressources qu'il recèle. 
On venait de très loin, de toute part, pour s'approvisionner, St-Augustin, Mingan, Goose Bay, Sept-Îles, Fort-Chimo et ailleurs [...] Oui, on se retrouvait nombreux en ces lieux. Ces chasseurs se désignaient comme les «Indiens du désert » car ils vivaient sur une terre sans arbre: la toundra. Leur rôle était de veiller sur les caribous, de savoir le nombre des prises annuelles; en terme français, ils faisaient donc office de garde-chasse (André 1984, 117).

Il reprendra, juste un peu plus loin: "Depuis infiniment longtemps, nous savions l'art de la conservation afin qu'aucune espèce ne fût menacée d'extinction» (André 1984, 118).

Dans son livre portant sur Les Innus et le territoire, Lacasse définit le rôle des Innus par rapport au territoire en termes de responsabilité et de gardiennage (2004, 218). Gouvernance et gardiennage innus: Innu tipenatimun, Innu kanauenitam. C'est ce double rapport à une gestion du territoire et à son rôle d'élément de permanence entre les générations qui se succèdent que nous retrouvons dans cette affirmation: "Nos ancêtres nous ont laissé ce territoire pour que nous l'utilisions et que nous le respections » (Nation innue 1997, 28).

De plus, le territoire est essentiel à l'Innu parce que la chasse constitue pour lui un mode de vie:

Autrefois, nos jeunes étaient très vaillants, ignorant l'oisiveté [...] Ils adoraient chasser et découvraient chaque jour de nouveaux territoires. Las du connu, ils partaient aisément en quête de nouveaux espaces pullulant de gibier recherché [...] Voici l'une des raisons pour laquelle nous pleurons nos territoires perdus car c'était cela notre gagne-pain: chasser dix mois par an! (André 1984, 115)

Mathieu Mestokosho parle de cette même chasse en ces termes:

Les Blancs en arrachaient plus que nous. Ce n'était pas leur travail. Ils trappaient mais ne savaient pas chasser. Chacun son travail. Dans le bois, le Blanc s'inquiète constamment, il s'affaiblit à propos de rien. La marche l'épuise. Il n'est pas chez lui. Pour l'Indien, c'est le contraire. Il est chez lui. Il sait chasser et il chassait toujours. C'était difficile, mais on s'en sortait par nous-mêmes. Nous étions fiers de ce travail, de notre chasse (Bouchard 1977, 100).

Mathieu André parle lui aussi de son sentiment d'appartenance au territoire: "Arrivé sur notre territoire, on se sentait chez-nous avec de la nourriture en abondance, nous étions heureux dans notre milieu» $(1984,43)$. Mestokosho souligne ici un autre caractère essentiel du territoire: c'est le lieu par excellence où l'Innu se sent à l'aise. 
En fait, les éléments spécifiques de la culture innue renvoient au territoire: la perception de soi comme élément d'un tout plus global qui donne sens à la vie de l'humain et en définit le rôle; le sentiment d'une nature généreuse qui fait vivre l'humain et fournit un support à son partage avec les autres êtres. C'est au territoire que se rattache toute la compréhension innue du monde. Ce rôle clé du territoire sera évoqué en ces termes par ceux qui relèvent de la culture innue: "C'est le territoire qui donne un sens à tout le reste: tout y est relié et c'est ce qui rend cohérent l'ensemble de nos activités »(Mamit Innuat 1986, 162; Lacasse 2004, 152). «Nitassinan fonde notre identité de peuple Innu et nous oblige à la protéger pour assurer notre avenir. Sans Nitassinan nous ne saurions survivre comme peuple » (Innu takuaikan 1990, 18 ; Nitassinan désigne le territoire innu).

Mais sur quoi cette importance du territoire est-elle fondée ? Qu'est-ce qui porte l'Innu à se considérer comme partie prenante de la nature qui l'entoure et à vouloir s'y insérer plutôt que de chercher à la dominer ? Où puise-t-il le sens de sa responsabilité envers les lieux qu'il fréquente, alors que l'extinction des ressources et la pollution ne suffisent pas à contraindre les gouvernements des pays occidentaux à des mesures qui iraient à l'encontre d'une rentabilité économique immédiate? Qu'est-ce qui rend l'Innu plus à l'aise sur son territoire dans le bois que dans n'importe quelle maison, si confortable soit-elle? Qu'est-ce qui lie la spiritualité aux territoires de chasse et qui n'est pas transmise par la religion apprise des missionnaires?

Ces questions nous incitent à pousser plus avant notre réflexion et à interroger les fondements mêmes de la conception innue du monde.

\section{La perception du monde}

Pour les Innus, le monde renvoie au sacré, il y renvoie même doublement et cette perception se fonde sur une vision de l'être. Comment comprendre cette double sacralité du monde appuyée sur une conception de l'être?

En premier lieu, nous verrons comment tous les éléments du monde renvoient à une assise spirituelle qui leur est propre, comment le monde est habité par le sacré et comment il est rempli de forces spirituelles.

Ensuite, nous examinerons comment cette perception de la présence d'un fondement sacré à chaque élément du monde suscite une vision active du rapport au monde et au sacré qui l'habite: l'humain doit créer des relations de don avec les éléments du monde et cela lui permet à la fois de soutenir sa vie et de perpétuer celle du monde. 


\subsection{L'assise sacrée de tous les êtres}

Le monde qui se présente dans sa réalité visible se manifeste en même temps comme une présence dont il est habité. Le monde s'offre comme vérité à déchiffrer à partir de tous les êtres qui en composent la réalité. Don de ce qui est là et qui fait sens parce que sa manifestation en soutient l'évidence, parce que quelque chose soutient son apparition, qui invite à le considérer dans l'accomplissement de son être qui apparaît comme plénitude. Le monde est don, don de l'être qui s'offre comme sens à accepter d'abord et à constamment interroger pour en approfondir la densité. Le monde fait sens parce qu'il s'appuie sur une assise invisible, une force qui en définit et en supporte l'être.

Chaque réalité du monde s'offre donc comme une plénitude disponible. L'idée d'un support invisible ou spirituel adapté à chaque réalité et lié constitutivement à elle correspond à cette autonomie de chaque être dont la présence se donne avec le monde. La réalité qui entoure les humains se propose comme la clé du sens à partir de laquelle ils peuvent se comprendre et interpréter le monde. Celui-ci n'est pas d'abord rapporté à des dieux mais à une multitude d'assises sacrées qui se situent au fondement de chaque entité et la font être ce qu'elle est. C'est aussi le support sacré de chaque être qui le justifie dans sa façon d'être et qui en fait une réalité complète et parfaite en elle-même. C'est un sacré à chaque fois particularisé comme force ou puissance singulière qui sous-tend chacun des êtres tel qu'il se présente et qui rend compte de l'ordonnance du monde. Ainsi, l'invisible - on pourra parler aussi de spirituel et Le Jeune parlera d'âme (Relations, 1634, 16 ) — soutient le visible et lui est lié à l'intérieur même de chaque chose dans la singularité de sa manifestation. Tout le réel est donc constitué d'une double valence visible/invisible. Leur unité est si totale qu'on peut pratiquement dire que toute réalité est manifestation de l'invisible sur lequel s'appuie son être. Chacune des réalités du monde mérite donc considération et respect puisqu'en elle se dévoile un élément du sacré irréductible aux autres et qui la spécifie dans son être singulier.

Dans un tel monde, aucune des réalités n'est ramenée à un concept abstrait, à un genre dont chaque individu ne serait qu'un représentant, perdant là toute référence directe au caractère unique de sa présence. Dans la langue montagnaise, il n'y a de désignation que spécifique et donc relative: «ton père ", "ma mère ". Le point de vue qui prévaut ici est celui de la particularité et de la relation. Nous reviendrons d'ailleurs sur ce dernier point de vue qui est fondamental dans l'analyse innue du monde et affecte 
la globalité de la réflexion qu'elle porte sur l'ensemble des êtres. Cette appartenance d'un élément invisible à tout élément du visible et qui le définit comme réalité unique est perçue en termes de force, de puissance spirituelle. Tous les éléments du monde recèlent ainsi une puissance spirituelle: humains, animaux, arbres, rivières, poissons, vent, etc. Il s'agit pour l'humain de la reconnaître dans son incidence propre et de négocier avec chacune d'elles une collaboration possible.

L'Innu vit dans un monde dont chacune des composantes est en soi porteuse d'un élément particulier et donc incontournable du sens. La compréhension du monde implique de prendre en compte la multiplicité des êtres qui sont tous porteurs de signification et de les intégrer à une structure commune du sens qui fait place à chacun des éléments qui le composent.

À l'intérieur d'une telle compréhension du monde, nul besoin de faire appel à une réalité sacrée qui soit extérieure au monde pour en asseoir les fondements. Le sacré, lié d'une manière unique à chaque constituant du visible, habite le monde. Toute réalité et spécialement celle qui entoure le quotidien de l'humain réfère à une assise spirituelle et peut donc la dévoiler à celui qui y est attentif. Ce monde est celui où toute réalité visible est tellement liée à son support invisible que celle-ci est susceptible d'en dévoiler la présence à tout moment.

Le monde est perçu comme une totalité qui inclut tous les êtres qui le constituent. Dans un tel monde, le temps n'est pas d'abord perçu comme linéaire et orienté vers un ailleurs, tel qu'il en est pour le nôtre. Le donné, appuyé sur le sacré dans son être, n'a pas à devenir autre que ce qu'il est. Le moment essentiel est celui qui a défini le monde tel que les humains le connaissent: c'est celui d'un passé où les ancêtres primordiaux ou les héros culturels l'ont organisé selon les modalités qu'il possède maintenant. LéviStrauss parlera en ces termes du rapport temporel aux événements fondateurs que rapporte le mythe: "la valeur intrinsèque attribuée au mythe provient de ce que ces événements, censés se dérouler à un moment du temps, forment aussi une structure permanente. Celle-ci se rapporte au passé, au présent et au futur» $(1958,231)$.

Dans une telle conception du temps, les péripéties de l'histoire se rapportent les unes aux autres sans que leur antériorité ou leur postériorité ne constitue une clé d'explication ainsi qu'il en est dans la tradition occidentale où «l'avant détermine l'après, et la série de ces déterminations est irréversible» (Eco 1993, 121). Ce n'est pas ici la chaîne des causalités appuyée sur l'antériorité de l'événement qui intéresse, puisque ce n'est pas 
elle qui fait sens (Vincent 2002, 103). On cherche plutôt à mettre en rapport deux événements qui présentent une analogie. Ainsi, une situation actuelle mise en relation avec une occurrence correspondante du passé pourra permettre de l'interpréter tout en prenant elle-même sens dans ce rapprochement (Vincent 2002, 103). La vraie causalité créatrice est celle qui a eu lieu au moment où s'est constituée l'organisation du monde, quand les ancêtres fondateurs lui ont donné la figure que nous lui connaissons: maintenant, c'est dans leur rapport les unes aux autres, que les choses prennent sens. En fait, dans une telle appréhension du réel, toutes les choses sont présentes les unes aux autres, elles sont imbriquées les unes dans les autres et s'interpénètrent. Dans une telle conception du temps, tout peut renvoyer à tout.

De même, les frontières entre le visible et l'invisible sont perméables: ainsi, un héros culturel bien connu des Innus, Tsakapesh (Lefebvre 1974, 21-32), n'a qu'à désirer voir le réel prendre telle direction pour voir s'accomplir son désir. Mais il s'agit bien sûr du temps primordial... Pourtant, les chamans d'un temps à peine antérieur pouvaient concentrer leur pensée sur leur désir et le voir s'accomplir par la force de leur correspondant spirituel (Speck 1977, 191). Comme Mathieu André en a fait la remarque, «il y a des récits que l'on se raconte de génération en génération depuis si longtemps que la frontière entre le naturel et le surnaturel est devenue impossible à retrouver, de telle sorte que certaines histoires vraies vous paraîtront extraordinaires même si elles ont pour origine des faits réellement vécus» $(1984,1-2)$. Et il citera plus loin une de ces histoires qui nous paraissent extraordinaires concernant des êtres invisibles, mais qu'André tient à situer dans sa relation avec un temps historique: "On raconte qu'autrefois il pouvait arriver qu'une jeune fille épousât un Homme-crapaud [...] Il s'agissait d'êtres invisibles ». Et de raconter comment une jeune fille amoureuse d'un homme-crapaud qu'elle seule pouvait voir est enlevée par lui et devient sa femme et comment son père sera approvisionné en caribou par son gendre invisible. Et de conclure: "Ce n'est pas une légende mais une histoire vraie. Elle est ancienne bien que le magasin de l'Hudson's Bay Co. du lac Petissikupau fût déjà en service. On la doit aux temps de l'existence des chamans" (André 1984, 39). Histoire vraie, mais qui relève de l'autorité des chamans capables de faire communiquer les mondes visibles et invisibles...

L'être humain partage avec les autres éléments du monde une même structure d'être. Lui aussi est constitué d'un être extérieur visible et d'une 
réalité spirituelle personnelle invisible — son Mistapeu (Vincent 1976, 24) - avec laquelle il travaille toute sa vie à approfondir une relation qui lui permettra tant d'avoir des succès à la chasse que d'atteindre un développement maximal de sa personnalité et ainsi jouer adéquatement son rôle social. C'est aussi à l'intérieur du lien à son Grand Homme (c'est ce que signifie Mistapeu) qu'il pourra entrer en contact avec les différents éléments de la réalité spirituelle qui sous-tend le monde et qu'il saura poursuivre la quête spirituelle qui constitue un objectif essentiel de sa vie. Il est donc, parmi toutes les autres, une réalité qui participe d'un sacré susceptible d'entrer en communication avec les autres éléments sacrés constitutifs de l'univers. L'humain se perçoit ainsi comme un parmi d'autres. Sa vie est liée à son appartenance et à sa participation au monde, mais cette participation est d'ordre spirituel autant que physique. Il adhère à un imaginaire d'inclusion dans le monde: le monde est une réalité qui l'englobe et c'est en s'insérant dans la totalité qu'il constitue que l'humain pourra prendre part à la vie qui l'anime. La vie de l'humain - comme celle de tous les êtres dépend de son incorporation à l'ordre d'un monde qui est conçu à la manière d'un système complet en soi. C'est en ce sens que nous avons évoqué plus haut une totalité pour parler de la perception innue du réel: un monde formant un ensemble défini par tous les éléments qui le constituent. Ceux-ci, en étant fondés sur des assises spirituelles qui en assurent la perfection hors de tout mouvement pour tendre vers autre chose, constituent un univers où tout s'est établi au commencement, atteignant d'un coup la complétude de ce qu'il a à être et où tous les gestes s'orientent vers une reprise d'un donné selon les modes définis par les héros culturels.

Nous nous situons donc ici dans un imaginaire de la continuité et de la participation. Le cosmos, la société, les conditions de vie sont d'abord à recevoir tels qu'ils se donnent. L'humain se perçoit plus comme inclus dans une réalité qui le porte et dont il reçoit la vie que comme celui qui agit sur le réel pour le soumettre à sa domination. Son effort tend vers une intégration dans le monde plutôt que vers une maîtrise de celui-ci et son attitude envers lui est faite de respect et de reconnaissance.

\subsection{Un rapport actif au monde: les dons entre les êtres}

Mais pour concevoir le monde comme une totalité impliquant des réalités toutes singulières, il faut faire appel à un principe susceptible de les coordonner entre elles, parce qu'autrement un tel ensemble s'avèrerait complètement statique et incapable de soutenir l'avancée d'aucune vie, puisqu'il n’y 
aurait rien pour le mettre en mouvement. Comment des réalités appuyées sur des puissances tout individualisées et parfaitement autonomes pourraient-elles créer un monde si elles ne se risquaient à entrer en relation les unes avec les autres? Comment pourrait-il y avoir surgissement de vie si chacune des réalités en restait à son espace de définition autosuffisante ? Il n'y aurait pas de monde à proprement parler, mais une parcellarisation d'identités différentes les unes des autres et incapables de communiquer entre elles. L'imbrication visible/invisible à l'intérieur de chaque être suppose donc déjà, pour sa complétude, un autre principe qui rende compte de la capacité du monde à renouveler la vie en son sein; un tel rôle est joué par le don. C'est à l'intérieur du lien créé par le don entre les êtres d'un même groupe et par ceux qui ont lieu entre les différentes catégories d'êtres que l'unité peut se constituer et qu'advient le mouvement qui permettra à la vie de se poursuivre. Ainsi, c'est par le don que les animaux font de leur vie aux humains que ces derniers peuvent poursuivre la leur: en retour, ceuxci rendront reconnaissance et respect aux animaux pour la vie qu'ils en ont reçue et traiteront leurs restes de façon à ce qu'ils puissent revenir à la vie. Parce que si rien ne se produisait entre les êtres, si chacun en restait à l'unité visible/invisible qui le définit, il n'y aurait précisément pas de monde: seulement une multitude de réalités sans organisation et sans prise les unes sur les autres.

L'accessibilité du monde à l'humain suppose donc que celui-ci puisse nouer des relations avec les différents êtres qui le constituent pour entrer en relation d'alliance avec eux. Le cosmos s'avère vie envers celui qui peut obtenir d'autres êtres ce qui pourra soutenir son existence en suscitant avec eux des rapports de dons et contre-dons. Ce sont les dons que se font entre eux les différents groupes d'êtres qui mettent en rapport les forces individuelles qui sont à la racine des différentes réalités et organisent celles-ci en un tout cohérent. La trame du monde se constitue à partir des réseaux de liens que créent entre elles les différentes réalités. Ces dons qui ont lieu d'un groupe à l'autre, en plus de rendre possible la poursuite de la vie pour chacun, permettent aussi au monde de poursuivre plus avant son existence. L'alliance qu'ils créent entre les divers éléments s'avère donc être au fondement de la vie de chacun des êtres qui le constituent, comme au fondement de la permanence de celui-ci dans son ensemble.

Par le don, par l'échange entre les groupes d'êtres qui posent les uns visà-vis des autres des gestes d'alliance réciproque — selon les trois moments décrits par Mauss dans son Essai sur le don: donner, recevoir et rendre 
$(1965,205)$ - , se constitue et se perpétue l'univers. À partir de ces dons tour à tour reçus et rendus, chacun des êtres se situera au centre d'un réseau de relations où chaque geste posé trouve sa contrepartie. Le mouvement, plutôt que de tendre vers l'ailleurs d'un progrès infini comme dans la culture occidentale, se situe à l'intérieur de la totalité constituée à partir des liens et de l'équilibre qu'elle établit entre les entités qui la sous-tendent. Dans le don, tout circule, tout prend la place de tout, mais tout peut aussi revenir à son point de départ comme dans le kula des Trobriandais dont nous parle Mauss $(1965,176-180)$ où, circulant constamment entre les îles, les uns dans un sens, les autres en sens inverse, colliers et bracelets de prestige décrivent un cercle avant de revenir à leur point de départ. Image parfaite au niveau du monde des îles de la totalité que constitue l'aperception du monde dans les civilisations du don. La recherche de l'équilibre dans la mouvance des éléments prévaut ici sur l'avancée du temps. L'imaginaire est celui du cercle, de la totalité que constituent les différents éléments qui sont en interrelation constante les uns avec les autres et non pas, comme dans la civilisation occidentale, celui d'un axe du temps projeté vers l'avant en une avancée indéfinie. On pourra parler d'une compréhension circulaire du monde (Sioui 1994, 224-225).

En entrant en relation avec la multitude des réalités constitutives du monde, l'humain instaure un réseau de relations au centre desquelles il se situe. Et par la multiplicité des réseaux constitués à partir de chacune des réalités, se crée une socialité globale, incluant non seulement les humains mais aussi tous les êtres. L'unité du monde dont le sens se donne à partir de chacune des parties suppose cette sociabilité intense créée par les différents réseaux de relations qui s'établissent entre tous les êtres. Chacun se définit par les relations qu'il a aux autres. Plus on a de liens et plus on est reconnu. L'individu se trouve ainsi au centre d'un réseau de relations de don avec les différents groupes d'êtres qui l'entourent, notamment avec les animaux qu'il chasse. Et plus les humains partagent entre eux et entretiennent de liens de don avec les autres groupes d'êtres, plus se renouvelle la vitalité du monde.

Le rapport aux différents éléments du monde est aussi pour le chasseur un moyen d'approfondir sa connaissance de l'élément spirituel du monde et de celui sur lequel se fonde son être, son Mistapeu. En effet, la chasse implique d'abord le développement d'une relation suivie avec le soi spirituel de l'humain qui doit être attentif à toutes ses suggestions - manifestées essentiellement dans les rêves - et doit accomplir la chasse des 
animaux qu'il a obtenus pour lui du monde spirituel. Parce que la chasse - et plus largement toute relation avec un des éléments de la nature - se joue largement au niveau spirituel. C'est la constituante spirituelle de l'humain, son Mistapeu, qui négocie avec les entités spirituelles responsables des animaux les prises qui lui sont dévolues. Ainsi, en chassant, l'humain subvient à ses besoins et aux besoins de sa famille; en même temps, il approfondit sa connaissance de la réalité spirituelle présente à tous les éléments du monde. Pour chasser, il joue du tambour et chante les chants que les maîtres des animaux lui ont suggéré en rêve, et ainsi il pourra entrevoir en songe l'animal qu'il pourra prendre. Et sa connaissance du territoire est tellement profonde que souvent il pourra situer le lieu de la forêt où doit avoir lieu sa rencontre avec l'animal et s'y rendre pour accomplir sa chasse. C’est ainsi que Mathieu Mestokosho, dans le récit des déplacements qu'il accomplissait annuellement entre Mingan et North West River au Labrador rapportera avoir tué un caribou que d'autres avaient en vain essayé de prendre après l'avoir entendu chanter sur un lac dans son rêve. Le lendemain, il abattra l'animal sur ce lac qu'il avait vu en rêve en ayant nettement conscience que c'était à lui que ce caribou était réservé (Bouchard 1977, 120).

Le don constitue essentiellement une reconnaissance de l'autre qui s'appuie sur une affirmation de soi. Celui qui donne veut honorer son partenaire au moyen d'un objet, d'une prestation ou d'un service par lequel il le reconnaît et en même temps s'affirme en tant que sujet qui provoque la réponse de l'autre. Si, comme l'affirme Mauss dans son Essai sur le don, « on se donne en donnant» $(1968,161)$, on voit à quel point la proposition d'alliance constituée dans le geste du don constitue un risque : c'est quelque chose de soi qui se porte vers l'autre en le mettant au défi de se compromettre de la même manière envers soi. Risque dont l'enjeu est l'établissement du lien social et d'une alliance pratiquement définitive entre les partenaires. Ce déplacement du soi dans le domaine de l'autre qui appelle la réciproque établit la présence de chacun au centre d'un multiple réseau de relations entre tous les êtres. Relations qui intègrent le cosmos tout entier dans la trame que constituent les relations de dons qui rapprochent entre elles les différentes catégories d'êtres qui se reconnaissent réciproquement et créent des liens. C'est ce mouvement de rapprochement réciproque que décrira ainsi Hénaff $(2002,171)$ : «cela constitue l'avancée du même au cœur de l'autre ou de l'autre au cœur du même. Chacun est au centre d'une toile tissée de ces liens nombreux. Ainsi s'engendre une sorte d'ubiquité réciproque qui surmonte le paradoxe du tout et des parties, de 
l'un et du multiple, du ici et du là-bas, du local et du global. C'est bien cela que l'on peut appeler le lien social ». Précisons ici que le lien social concerne l'entièreté des êtres et non seulement les humains.

Ces différentes réalités constituent un ensemble, dans la mesure où tout bouge dans le don et où tout est ainsi imbriqué, tout est interpénétré. La réalité, au départ statique et multiple peut ainsi se constituer en un tout dont la vie pourra se reproduire et perdurer. Dans son étude portant sur les Kwakiutl de la Côte Ouest - mais il pourrait tout aussi bien parler des Innus - Goldman affirmera $(1975,124)$ :

L'obligation de réciprocité dans l'échange n'est pas une réponse à des pouvoirs spécifiques liés aux objets mais une conception cosmique qui présuppose une circulation éternelle des espèces et des êtres. Les obligations de donner et de rendre sont des obligations de participer à cette circulation vitale [...] Les chefs kwakiutl sont partie prenante d'échanges où ils incarnent des ancêtres et des êtres surnaturels. Pour eux, donc, le système total des échanges englobait un univers peuplé d'hommes, d'esprits ancestraux, d'êtres surnaturels et, à travers les biens mis en circulation, un univers de formes de vie animales et végétales.

Le spirituel accorde des dons à l'humain, dans la mesure où celui-ci partage avec les autres êtres et fait preuve de générosité et d'entraide envers ceux-ci. Ainsi, le chasseur qui s'est montré respectueux envers l'animal tué, qui a traité ses restes de façon à ce qu'il puisse revenir à la vie, qui partage la nourriture avec ceux qui en manquent, s'attendra à ce que l'esprit maître de l'animal continue de lui allouer du gibier. C'est pourquoi les festins sont si importants dans la culture traditionnelle innue : c'est en partageant qu'on pourra recevoir encore. Ce sont les dons qui font circuler la vie et l'énergie. Le don met en branle toutes les forces du monde et stimule le cosmos dans son ensemble. Plus les êtres humains partagent entre eux, plus il y a d'échanges entre les différents groupes d'êtres, plus chacun recevra et aura à partager. Le cosmos tout entier est stimulé par les dons qui se font entre les êtres (Ingold 2000, 114).

La langue innue reflète d'ailleurs cette prédominance de la relation dans la culture. Le système verbal ne se développe pas à partir de la notion de temps, mais à partir du lien de l'action avec les choses qui l'entourent, c'est-à-dire du verbe avec ses compléments. Chaque nouvel état de la relation avec les choses suscite une nouvelle unité verbale. Le système de la langue manifeste ainsi la centralité du lien. 


\section{Pistes...}

Le regard que les Innus portent sur le monde, le rapport au territoire, l'organisation sociale et les valeurs diffèrent largement de ceux de la société occidentale. Ce que nous avons voulu montrer ici, c'est qu'elles relèvent d'une logique qui s'avère aussi cohérente et défendable que celle qui soustend cette dernière et que la disparition d'une telle culture constituerait une perte irréparable au plan du patrimoine humain. La question qui se pose pour nous maintenant pourrait se formuler ainsi: Sommes-nous prêts à admettre la présence d'une autre culture à l'intérieur de notre société et d'en respecter les assises, afin qu'elle puisse être en mesure de développer son expression? Voulons-nous encore par tous les moyens exercer des pressions pour constituer une société monolithique comme cela s'est fait dans le passé ou pouvons-nous admettre une vraie collaboration avec une culture différente de la nôtre? N'y a-t-il d'autre alternative que le melting pot ou les «camps de réfugiés " (Savard 1979, 63), l'assimilation pure et simple ou l'exiguiité des réserves? Nous venons de souligner comment la relation au monde des Innus comportait une double relation au sacré dans la culture traditionnelle: 1) reconnaissance de l'élément spirituel qui habitait chaque être et en fondait l'irréductible individualité et la perfection; 2) entrée de l'humain en relation de don et de contre-don avec les éléments du cosmos pour soutenir sa vie et permettre en même temps au grand tout que constitue le monde de poursuivre la sienne. À l'intérieur de la vie de tous les jours, relation au monde implique relation au territoire; dans la pratique, le monde est le territoire. C'est cette double notion de la sacralité du monde qui suscite l'attitude fondamentale d'inclusion des humains à l'intérieur des éléments du territoire, de l'organisation de celui-ci en fonction d'un partenariat entre humains et non-humains et du gardiennage dont ils reconnaissent la responsabilité qui caractérise les Innus.

Par ailleurs, nous savons aussi que les deux regards portés sur le monde, celui de la culture occidentale et celui de la culture innue, doivent en arriver à des compromis nécessaires pour qu'ils puissent cohabiter. Nous ne pouvons plus vraiment continuer à refuser de reconnaître aux Innus le droit d'affirmer leur culture et de disposer d'une plate-forme viable pour en manifester l'expression. Et voudrait-on le faire qu'on se heurterait tôt ou tard à ce qui leur a été reconnu par la constitution canadienne. Nous savons en effet que celle-ci reconnaît aux Premières Nations des droits sur le territoire qu'elles utilisaient avant l'arrivée des Européens et qu'elles ont continué à utiliser depuis lors. Persiste évidemment la question de savoir ce 
qu'implique la notion d'utilisation continue, mais les tribunaux sont là pour le déterminer. Ce que nous savons toutefois, c'est que l'existence des Innus en tant que peuple possédant une culture propre est liée à la possession d'un territoire qu'ils pourraient gérer à leur manière (Innu aitun) et où ils pratiqueraient un mode de vie conforme à leurs valeurs, leur culture et leur spiritualité. Sommes-nous prêts à leur reconnaître le droit à l'existence comme peuple?

Par ailleurs, ce qui en premier lieu doit se comprendre comme un simple respect de l'autre dans sa différence et comme acceptation de sa culture pourrait aussi s'avérer bénéfique pour nous. Notre société en arrive en effet à expérimenter d'elle-même les limites du modèle qui la sous-tend, celui d'un développement sans limites, celui de la domination de l'être humain sur le monde qui fait de celui-ci un pur instrument dont il dispose pour satisfaire n'importe quelle envie. Beaucoup de ceux qui partagent la culture occidentale croient que le développement sans fin des appétits de l'humain à partir du pillage de la nature n'est plus possible et que l'on doit revenir à une gestion des ressources qui tienne compte de la capacité de la nature à se renouveler. N'y aurait-il pas moyen, en reconnaissant le droit à l'existence d'une autre culture - ce qui implique une assise territoriale sur le Nitassinan et une autonomie gouvernementale qui permette aux Innus d'organiser la collectivité qu'ils constituent selon leur propre cadre juridique et leurs propres valeurs - de nous enrichir des éléments qu'une autre conception du monde pourrait apporter pour suppléer aux carences de la nôtre?

On pourrait ainsi penser à nouer avec le monde un rapport qui ne se constitue pas seulement à partir d'une volonté de domination. Peut-être les problèmes que nous affrontons actuellement proviennent-ils en partie du refus de l'humain de s'inclure parmi les éléments de la nature et de sa décision de ne la considérer que comme un objet qui doit se plier à son vouloir. L'occultation de son inclusion dans la nature constitue en effet pour l'homme une perte qui a eu des conséquences graves; selon Lévi-Strauss $(1973,53)$ :

On a commencé par couper l'homme de la nature, et par le constituer en règne souverain; on a cru effacer ainsi son caractère le plus irrécusable, à savoir qu'il est d'abord un être vivant. Et, en restant aveugle à cette propriété commune, on a donné le champ libre à tous les abus [...] en s'arrogeant le droit de séparer radicalement l'humanité de l'animalité, en accordant à l'une tout ce qu'il retirait à l'autre, il (l'homme occidental) ouvrait un cycle maudit et la même frontière, constamment reculée, servirait à écarter des hommes d'autres hommes, et à revendiquer, au profit de minorités toujours 
plus restreintes, le privilège d'un humanisme, corrompu aussitôt né pour avoir emprunté à l'amour-propre son principe et sa notion.

Cette inclusion de l'humain parmi les éléments du monde ne permettrait-elle pas d'entretenir avec lui un rapport moins centré sur le profit? Des textes déjà anciens mettent bien le doigt sur les différences entre les attitudes des deux cultures face aux ressources du territoire et ils nous laissent mal à l'aise quand nous voyons comment on caractérise les comportements de ceux qui appartiennent à notre culture. Ainsi, le Superviseur des terres à bois indiennes affirmera en 1926:

La comparaison entre le mode de piégeage indien et celui du trappeur blanc moyen prouve en dehors de tout doute que l'Indien est un conservationiste. Les Indiens trappent sur les mêmes terres chaque année, récoltent les fourrures au moment où elles sont à leur qualité maximale, s'abstiennent d'exterminer totalement les animaux à fourrure et restreignent prudemment leur usage du feu en forêt.

Le trappeur blanc moyen n'a pas souci d'avoir continuellement à effectuer son piégeage dans le même district. S'il trouve un endroit prometteur, son objectif est de l'épuiser en une saison, pour changer de région l'année suivante. Avec cela à l'esprit, il recourt à des méthodes dont le résultat est l'extermination des populations d'animaux à fourrure dans tout son territoire (RG 10, vol. 6750. Filière 420-10A, bobine C-8106; cité par Scott et Morrisson 2005, 42).

Peut-être le sentiment d'appartenance à la terre aiderait-il l'homme occidental à retrouver cette émotion devant la générosité du monde à son égard: impression d'être entouré d'une abondance qui ne demande qu'à se donner à lui pourvu qu'il partage avec d'autres ce qu'il a reçu, sentiment de co-appartenance à une même réalité et de respect face aux différents éléments du monde qui forment son milieu et lui procurent la vie. N'est-ce pas cela que l'on recherche à travers toutes les formes de tourisme qui visent à nous remettre en contact avec la nature ?

Revenons maintenant à notre question du début : pourquoi distinguer entre religion et spiritualité et rapporter l'une au village et l'autre à la présence dans les territoires? Qu'est-ce qui pourrait fonder cette distinction? Comment le territoire peut-il être présent à la spiritualité innue qui relève d'une culture traditionnelle et non de la religion qu'ils pratiquent quand ils sont sédentaires à la réserve? Nous croyons que cette distinction faite par nos interlocutrices innues n'était pas sans objet. 
Nous savons tous que la très grande majorité des Innus s'est convertie au christianisme et cela, pour une bonne part d'entre eux, dès les débuts de l'implantation française sur le territoire du Québec. Les Jésuites rêvaient de sédentariser les Amérindiens en les invitant à vivre dans des réductions où ils pourraient leur enseigner la foi chrétienne et leur apprendre l'agriculture. Invités à venir s'établir à Sillery pour être près des Jésuites, les Innus de Tadoussac insisteront plutôt pour que ces derniers viennent les rencontrer pour leur enseigner le christianisme au moment et au lieu où ils reviennent près du fleuve après avoir fait leurs chasses de l'hiver. Les missionnaires seront donc acculés à risquer la christianisation d'un peuple de nomades qu'ils ne peuvent rencontrer que pendant un court moment dans l'année (Relations, 1641, 52 ; Campeau 1979, 122-123). Cela aura comme conséquence une grande autonomie de ces derniers dans leur adoption de la nouvelle foi et la transmission qu'ils s'en feront les uns aux autres. Une étude du christianisme innu faite à partir des prières écrites dans leur langue et contenues dans un Livre de prières et de chants (Aiamieuna mak nikamuna), actuellement en usage dans les différentes communautés de la Côte Nord du Saint-Laurent — dont l'original a été publié en 1867 et dont on a fait depuis de nouvelles versions avec des ajouts en reprenant les sources anciennes -, nous montre une large part de continuité entre la spiritualité traditionnelle et le christianisme adopté par les Innus (Doran 2005, 322347). Continuité entre l'approfondissement du spirituel auquel les Innus consacraient leur vie et qui était lié à leur pratique de la chasse - mystique de la chasse, en quelque sorte (Speck 1977, 9) — et à leur recherche d'union à Dieu dans le christianisme; continuité encore entre leur appartenance à un système du don et le christianisme qu'ils ont compris à l'intérieur d'une telle perspective. La prière innue nous est même apparue comme fondée sur une énergétique de l'amour : l'amour de Dieu y est perçu comme l'origine de tout dynamisme qui ramène vers lui ses enfants. Nous retrouvons ainsi dans la prière des demandes du type "Aime-moi et je t'aimerai de tout mon cœur » ou des réponds aux litanies où un "Chéris-nous » remplace notre "Aie pitié de nous». Nous pouvons rapprocher ce que nous avons nommé énergétique de l'amour (Doran 2005, 136) de cette circulation des formes vivantes dont parlait Goldman et que nous avons mentionnée précédemment; ou encore du «don constant, débordant, infini » à l'origine de l'être dont parle Hénaff $(2002,268)$ : quelque chose surgit et circule qui est de l'ordre du don, qui suscite la vie et qui doit continuer pour que celle-ci persiste. En étant situés par leur culture traditionnelle dans une 
compréhension du monde où sont déjà donnés toute vie et tout ce qui est, où il s'agit de perpétuer ce don initial en créant des alliances qui font circuler la vie entre les partenaires - qui confirment par des dons l'importance de leurs alliés - les Innus ont pris pied dans un christianisme centré sur l'amour de Dieu qui a été à l'origine de tout ce qui est et auquel il faut encore se référer pour que la boucle puisse être bouclée. En créant et en maintenant l'humain à l'intérieur de la vie, Dieu lui a fait une proposition d'alliance qu'il renouvelle constamment. C'est en s'appuyant constamment sur ce don que l'humain peut espérer le faire circuler — nous nous situons encore à l'intérieur du cercle ou dans une totalité — jusqu'à ce qu'il revienne à son origine.

Pourtant, si cette compréhension du christianisme a pu situer la nouvelle religion qu'ont adoptée les Innus en continuité avec la compréhension de l'univers qui découlait de leur spiritualité traditionnelle, il est un point sur lequel la transition n'a pu s'opérer et cela à cause même des insuffisances de la pensée théologique qui sous-tendait l'enseignement qu'ils ont reçu. En effet, en culture traditionnelle, la perpétuation du monde s'opérait à partir des dons qui renouvelaient l'alliance des différents groupes d'êtres entre eux et la recherche d'approfondissement du spirituel par l'humain présupposait une relation constamment renouvelée avec les différentes catégories d'êtres. C'est donc dans son lien aux différents éléments du monde, qui se concrétise sous forme de dons - souvenons-nous que la chasse supposait une alliance entre le pendant spirituel de l'humain, son Mistapeu, et le maitre de l'animal pour constituer un rapport de don entre humain et animal —, que l'humain pouvait contribuer à la perpétuation du monde tout en subvenant à ses besoins. La poursuite de la vie, mais aussi l'approfondissement de la quête spirituelle de l'humain passaient ainsi par son lien avec les éléments constitutifs du monde. Or, cela ne se retrouve pratiquement plus $^{2}$ dans les prières chrétiennes innues. Le rapport au monde n'y est pas présent. Qu'est-ce à dire ? C'est que la théologie occidentale, enseignée essentiellement aux Innus par les Jésuites et les Oblats, ne contenait pas d'élaboration théologique consistante sur le rôle du monde à l'intérieur de la foi qu'elle proposait: le péché avait marqué l'échec de la création et la rédemption s'accomplissait pratiquement sans elle. C'est donc à cause des lacunes de notre pensée théologique que les Innus ont dû renoncer à cette part importante et tellement intéressante de leur culture en adoptant le christianisme. Et le plus regrettable, c'est que les fondements d'une telle

2. À une exception près : voir Doran $(2005,278-280)$. 
réflexion théologique existaient et avaient été posés à l'époque patristique. Quand un Origène disait, par exemple : "Le monde est plein de mystères » $(1981,3,8)$ pour signifier comment les éléments du monde physique ont une résonance divine depuis que le Verbe ressuscité a étendu les limites de sa corporéité aux dimensions de l'univers, il donnait au monde sa pleine capacité de dévoilement de la réalité spirituelle. Parce que la stature de ressuscité du Verbe ne connaît plus l'enfermement dans l'étendue restreinte de l'appartenance à un corps terrestre limité, elle peut assumer, récapituler en elle la plénitude de l'espace et du temps. Vision d'une totalité qui n'est pas sans rappeler cette conception du monde des Innus comme totalité à laquelle nous avons fait allusion et qui inclut un rapport au temps qui n'est pas linéaire. À l'intérieur d'un tel cadre de compréhension, tout contact avec le monde peut avoir une incidence sur la mise en relation avec la réalité christique, puisqu'elle est entrée en communication avec ce qui constitue son être de ressuscité. Il est temps que la théologie donne des assises aux implications spirituelles de la présence du monde au côté des humains, comme dimension intégrante de leur être spirituel autant que physique. Ne vaudrait-il pas la peine d'élaborer une théologie du monde pour que les Innus puissent trouver dans le christianisme les assises propres à supporter les intuitions de leur spiritualité traditionnelle et retrouver ainsi une continuité mieux établie entre leur réflexion antérieure et le christianisme qu'ils ont adopté ? Ne vaudrait-il pas la peine pour nous aussi d'approfondir la teneur proprement spirituelle de ce qui nous entoure, nous porte et nous façonne? Ne pourrions-nous pas même commencer à admettre qu'il y a dans les intuitions religieuses des autres cultures des éléments de réflexion dont nous aurions avantage à tirer profit ? Devons-nous croire que l'Esprit ne pourrait être à l'œuvre dans aucune culture qui chercherait à comprendre les fondements du réel hormis le judéo-christianisme? Admettre au départ que l'on pourrait trouver des éléments de réflexion stimulants dans les autres pensées religieuses ne pourrait-il pas contribuer à établir les assises d'un véritable œcuménisme et contribuer à enrichir la plateforme de notre réflexion religieuse?

Nous pouvons comprendre maintenant ce que suggérait la réflexion de nos interlocutrices du Musée de Mashteuiatsh. En liant la religion au village et la spiritualité au territoire, elles suggéraient que la sédentarisation et le christianisme se correspondaient parce qu'ils n'impliquaient pas à titre de référent essentiel le rapport au milieu de vie sur lequel s'appuyait la spiritualité traditionnelle. Tout un pan de la spiritualité traditionnelle innue 
était laissé de côté en christianisme: celui qui renvoyait l'approfondissement du spirituel aux liens qui se nouaient entre l'humain et les éléments du monde et qui avait été abandonné par la religion — comprenons le christianisme qui se vit en bonne partie dans une église. Cette spiritualité pouvait se déployer à son aise au village, parce qu'elle n'avait pas à y entrer en contact de la même manière avec l'environnement de l'humain. Pour retrouver le sens du vécu spirituel que connotait le lien avec la nature et plus largement le monde, il fallait se déplacer sur les territoires, là où l'environnement retrouvait la pleine dimension des mystères auxquels il donnait l'accès dans une mise en contact de l'humain avec les éléments de son environnement. La vie du village, comme le christianisme dont elle relevait, étaient moins prégnantes d'une spiritualité de mise en contact de l'humain avec son univers que les territoires.

\section{Références}

Aiamieuna mak nikamuna (1996), Shiship-pishim(u).

ANDré, M. (1984), Moi «Mestenapeu», Éditions Ino.

Bouchard, S. (1977), Chronique de chasse d'un Montagnais de Mingan. Mathieu Mestokosho, Québec, Ministère des Affaires culturelles du Québec.

Campeau, L. (1979), Monumenta Novae Franciae, II. Établissement à Québec (1616-1634), Rome/Québec, Les Presses de l'Université Laval.

Doran, A. (2005), Spiritualité traditionnelle et christianisme chez les Montagnais, Paris, L'Harmattan.

Dussault, R. et G. ERAmus (1996), Rapport de la Commission royale sur les peuples autochtones. 5 volumes. Ottawa, Groupe Communication Canada.

Eco, U. (1993), De Superman au Surhomme, Paris, Grasset et Fasquelle.

Gauchet, M. (1985), Le désenchantement du monde, Paris, Gallimard.

Goldman, I. (1975), The Mouth of Haven. An Introduction to Kwakiutl Religious Thought, New York, J. Wiley.

Heidegger, M. (1966), Lettre sur l'humanisme. Questions III, Paris, Gallimard.

Hénaff, M. (2002), Le prix de la vérité. Le don, l'argent, la philosophie, Paris, Seuil. 
Ingold, T. (2000), The Perception of the Environment. Essays on Livelihood, Dwelling and Skill, London/New York, Routledge.

InNu Takuaikan Uashat Mak Mani Utenam (1990), Vers une déclaration commune sur les droits du peuple québécois, du peuple canadien et des peuples autochtones, Mémoire présenté à la Commission sur l'avenir constitutionnel et politique du Québec, Sept-Iles.

Kapesh, A.A. (1979), Qu'as-tu fait de mon pays?, Ville Saint-Laurent, Editions Impossibles.

LACASSE, J.-P. (2004), Les Innus et le territoire. Innu tipenitamun, Québec, Septentrion.

Lamothe, A., A. Ashini et D. Vachon (1978), La vie sous la tente Patshuianitshuap e Apinanuti, Ateliers audio-visuels du Québec (Culture amérindienne-archives: document 68).

LAMOTHE, A. et R. LAPOINTE (1977), Linguistique montagnaise; Innu-Aimun, Ateliers audio-visuels du Québec (Culture amérindienne-archives: document 81).

LefebVRe, M. (1974), Tshakapesh. Récits montagnais-naskapi, Québec, Ministère des affaires culturelles.

Leroux, J. (1992), "Le tambour d'Edmond », Recherches amérindiennes an Québec, 22/2-3, p. 30-43.

LeVI-STrauss, C. (1958), Anthropologie structurale I, Paris, Plon. (1973), Anthropologie structurale II, Paris, Plon.

Mamit InNuat (1986), Rapport de consultation, Village-des-Hurons.

Mauss, M. (1968) [1925], "Essai sur le don, forme et raison de l'échange dans les sociétés archaïques ", Sociologie et anthropologie, Paris, Presses universitaires de France, p. 145-279.

Merleau-Ponty, M. (1945), Phénoménologie de la perception, Paris, Gallimard.

Origène (1981), Homélie sur le Lévitique. Tome 1 : Introduction et Homélies I-VII, trad. de M. Borret, Paris, Cerf.

Nation InNUE (1997), Ntsinan Nteeshinimitan Nteniunan (Notre terre, notre vie), Rapport du groupe de travail sur les activités minières, Sheshashit et Utshimassit.

Relations des Jésuites, 6 vol. (1972) [1858], Montréal, Éditions du Jour. 
SAVARD, R. (1979), "À la recherche d'une culture perdue... ", Sociologie et sociétés, 11/1, p. 57-63.

SCOTT, C. et J. Morrison (2005), «Frontières et territoires: mode de tenure des terres des Cris de l'Est dans la région frontalière Québec/Ontario II - Reconstruction et renouveau ", Recherches amérindiennes au Québec, 35/1, p. 41-56.

SiouI, G.E. (1994), Les Wendats. Une civilisation méconnue, Sainte-Foy, Presses de l'Université Laval.

Speck, F.G. (1977), Naskapi. The Savage Hunters of the Labrador Peninsula, Norman, University of Oklahoma Press.

VINCENT, S. (1976), "Les bonnes et les mauvaises alliances", Recherches amérindiennes au Québec, 6/1, p. 22-35.

(2002), «Compatibilité apparente, incompatibilité réelle des versions autochtones et des versions occidentales de l'histoire: l'exemple innu ", Recherches amérindiennes au Québec, 22/2, p. 99-106.

\section{Résumé}

À partir d'une comparaison entre l'attitude de l'Occidental face au monde qu'il considère avant tout comme un objet d'exploitation - et celle de l'Innu qui le perçoit comme un tout qui le porte et à l'intérieur duquel il doit se situer en relation d'alliance avec les autres éléments qui le constituent, cet article examine les fondements de la conception innue qui sont à l'origine de cette attitude: un monde dont chacun des éléments dévoile le sacré et à l'intérieur duquel tous les groupes d'êtres entretiennent des rapports de don. Il en conclut que pour les Innus, l'identité est liée au territoire. L'état québécois comprendra-t-il qu'une assise territoriale s'avère nécessaire à l'existence même de ce peuple et saurons-nous profiter de l'apport que sa conception pourrait apporter aux questions environnementales?

\section{Abstract}

Drawing on a comparison between the Western man's attitude towards the world - understood in terms of exploitable resources - and the Innu perception of the world as a totality which supports life and where he/she is inherently connected to other constitutive elements, this paper examines the foundations of the Innu conception of the world. All parts of this world are infused with and reveal the "sacred"; all the beings therein relate to one another through the notion of "exchange of gifts". The paper concludes that Innu identity is closely linked to the land. It asks whether the Quebec 
government is capable of understanding the need for a territorial basis to allow the continued existence of the Innu way of life. It also wonders about our collective ability to draw lessons from the Innu experience and conception of the land for the management of environmental challenges. 